\title{
Third order nonlinear optical response of PbS quantum dots
}

\author{
S. Chowdhury*, A.M.P. Hussain, G.A. Ahmed, D. Mohanta and A. Choudhury \\ Department of Physics, Tezpur University, Napaam, Tezpur, Assam-784 028, India \\ ${ }^{*}$ Corresponding author: e-mail: sky@tezu.ernet.in \\ Phone: +91-3712-231049
}

\begin{abstract}
PbS quantum dots of the average size $10 \mathrm{~nm}$ are synthesized in the polymer matrix (PVA) following chemical route. Optical absorption spectra reveal a large blue shift from the bulk absorption cutoff wavelength. Instead of using a high power laser, we have measured for the first time the third order nonlinear susceptibility $\left(\chi^{3}\right)$ of $\mathrm{PbS}$ quantum dots using a low power continuous wave laser with the Degenerate Four Wave Mixing (DFWM) technique. The result is encouraging in nonlinear optical application at room temperature.
\end{abstract}

Keywords: quantum dots, third order nonlinear susceptibility, DFWM.

Manuscript received 25.01.06; accepted for publication 29.03.06.

\section{Introduction}

Within the ever-expanding field of nonlinear optics the study of optical nonlinearity in semiconductor nanostructures such as quantum well or quantum dot is gaining importance due to their possible application in future high-capacity communication networks through the use of third order optical nonlinearities for ultrafast switching, signal regeneration, and high speed demultiplexing [1]. Materials with large third order nonlinear susceptibility $\chi^{3}$ thus have been an object of increasing interest in recent years. In particular, great effort has been devoted to the determination of the third order nonlinear optical susceptibility, $\chi^{3}$, responsible for phenomena such as third harmonic generation or optical phase conjugation $[2,3]$. Third order nonlinearities can be determined by many different processes, the commonly used are the z-scan technique, third harmonic generation and a large number of three and four wave mixing processes, the most common among them being the degenerate four wave mixing (DFWM).

Whereas in bulk semiconductors the physical origin of nonlinear absorption and refraction is associated with the optically created high density many particle system causing screening, band gap renormalization and band filling, the mechanism of nonlinearity in quantum dots is of a basically different kind. With decreasing size the optical nonlinearity is strongly influenced by quantum confinement.

By incorporating semiconductor nanoparticles into polymer, glass, or ceramic matrix materials, many of their interesting optical properties including absorption, fluorescence, luminescence, and nonlinearity may be studied. In these systems, very small particle sizes enhance the optical properties, while the matrix materials act to stabilize the particle size and growth. The strong confinement effect of the charge carriers can be precisely achieved in $\mathrm{PbS}$ system due to the large excitonic radius $(\sim 20 \mathrm{~nm})$ [4]. As the nonlinear optical properties of semiconductor quantum dots are expected to be greatly enhanced in the strong confinement regime, $\mathrm{PbS}$ is better suited for exploiting nonlinear properties.

Though the enhanced nonlinear optical properties of quantum confined semiconductors, attracted many, Wang et al. have been the pioneers in making these measurements on polymeric systems. In 1987, Wang and Mahler reported the first study of nonlinear optical properties in polymer stabilized CdS quantum dots using the DFWM [5].

There are several research papers reporting third order NLO susceptibilities of quantum dots. For CdS doped glasses Takada et al. [6] measured a $\chi^{3}$ value of $1.1 \times 10^{-6}$ esu $\left(1.5 \times 10^{-14} \mathrm{~m}^{2} / \mathrm{V}^{2}\right)$ while Woggon et al. [7] reported $\chi^{3}$ value of $3.2 \times 10^{-8}$ esu for CdS quantum dots. For surface modified CdS nanoparticle Yamaki et al. [8] observed $\chi^{3}$ value near $\sim 10^{-7}$ esu. For $\mathrm{PbS}$ quantum dots in PVA coating Lu et al. [9] measured a $\chi^{3}$ value of $1.06 \times 10^{-5}$ esu. Significantly some bulk semiconductor like $\mathrm{HgTe}$ possesses $\chi^{3}$ value as high as $1.6 \times 10^{-4}$ esu [10]. For DFWM experiment high power pulse laser like $\mathrm{Nd}$ :YAG or Ar ion laser are extensively used as the laser source [11]. Yang et al. [12] for the first time showed 
that a low intensity continuous wave laser can be used as the DFWM source. In their experiment they used an argon ion laser as the exciting light source. Earlier Kiessling et al. showed that phase-conjugated signals are achievable with relatively low intensities of the interacting waves (in the range $1-100 \mathrm{~mW} / \mathrm{cm}^{2}$ ) [13]. Recently Kurian et al. [14] studied optical nonlinearity induced in $\mathrm{PbS}$ nanoclusters by the z-scan method using low power continuous wave He-Ne laser. In this work, we report the first ever DFWM experiment using low power He-Ne $(\lambda=632.8 \mathrm{~nm})$ laser.

\section{Experimental details}

For chemical synthesis of $\mathrm{PbS}$ quantum dots in polyvinyl alcohol (PVA), we followed the method published elsewhere [15-18]. Typically, a $5 \mathrm{wt} \%$ PVA solution was prepared in double distilled water, by stirring in a magnetic stirrer with the rate $\sim 200 \mathrm{rpm}$ at a constant temperature of $70^{\circ} \mathrm{C}$ until a transparent solution is formed. To this solution $0.01 \mathrm{M} \mathrm{\textrm {PbCl } _ { 2 }}$ solution was added in the volume ratio $2: 1$ followed by stirring at the same rate and same temperature for three hours. To this solution, $0.01 \mathrm{M} \mathrm{Na}_{2} \mathrm{~S}$ was added drop wise, until the whole solution turns dark brown.

After keeping this solution in a dark chamber for 12 hours for stabilization, it was caste over glass substrate and dried. The film contains $\mathrm{PbS}$ quantum dots embedded in PVA matrix.

\section{Lead sulphide quantum dots}

\subsection{XRD study of PbS quantum dots}

Fig. 1 shows the XRD pattern of $\mathrm{PbS}$ quantum dots. For $\mathrm{PbS}$, the XRD peaks at $2 \theta=25.9^{\circ}, 30.1^{\circ}$ and $43^{\circ}$ corresponding to (111), (200) and (220) planes suggest formation of fcc crystal. The peak around $20^{\circ}$ corresponds to PVA crystalline phase. The average size of the prepared $\mathrm{PbS}$ samples was determined to be $\sim 12 \mathrm{~nm}$. The diffraction peaks are indexed according to the earlier published works [19, 20].

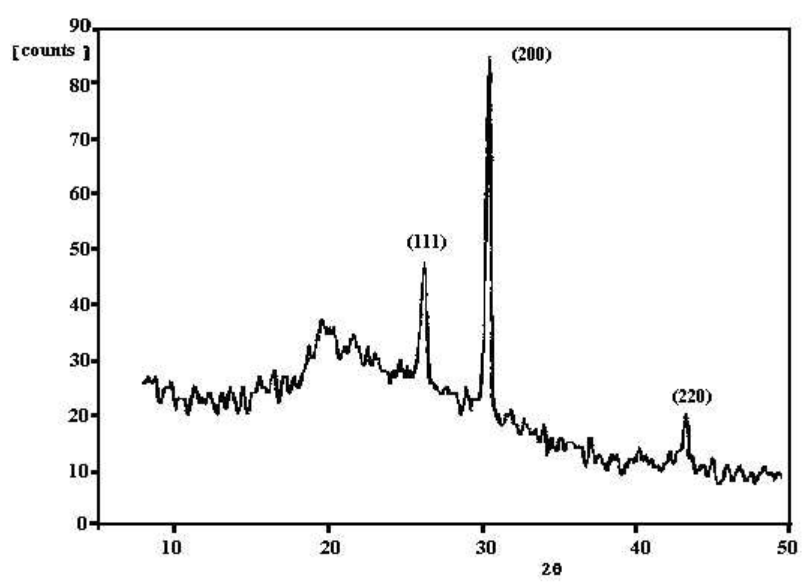

Fig. 1. XRD pattern of $\mathrm{PbS}$ quantum dots.

\subsection{UV-VIS optical absorption study}

The UV-VIS absorption spectra of $\mathrm{PbS}$ quantum dots are shown in Fig. 2. The absorption edge is found at $\sim 511 \mathrm{~nm}(2.42 \mathrm{eV})$ which is strongly blue shifted from the corresponding bulk value $(0.41 \mathrm{eV})$ indicating strong quantum confinement.

\subsection{Transmission electron micrograph study}

The transmission electron micrographs (TEM) display the pictorial view of size, shape and the surface morphology of the quantum dots. The TEM of PbS quantum dots embedded in PVA is shown in Fig. 3. The TEM image shows that the prepared $\mathrm{PbS}$ quantum dots possess nearly spherical shape with an average diameter of $10 \mathrm{~nm}$.

\section{DFWM experiment}

The experimental set up for DFWM is shown in Fig. 4. This is the counterpropagating-pump configuration. The wave from the laser source is divided into pump and probe waves by beam splitter $\mathrm{BS}_{1}$. The pump wave is further separated into two wave paths by beam splitter $\mathrm{BS}_{2}$, becoming pump waves $\mathrm{P}_{1}$ and $\mathrm{P}_{2}$. These two waves counterpropagate from both sides of the quantum dot sample $(S)$ and are injected into it. The probe wave $P_{r}$ reflected by the beam splitter $\mathrm{BS}_{3}$ is also injected into the sample along with the pump waves. The resulting phase conjugate wave is guided to the observation system by the beam splitter $\mathrm{BS}_{3}$. The amplified signal, detected with the help of a $\mathrm{Si}$ detector (TIL 81), is recorded with a storage oscilloscope [HM1507-3, HAMEG GmbH, Germany]. The sample was in the form of thin film measuring a thickness of $\sim 20 \mu \mathrm{m}$.

The $\chi^{3}$ value was calculated using the relation [9]

$$
\begin{aligned}
& \chi^{3}=\frac{4 n^{2} c \varepsilon_{0} \lambda \alpha \sqrt{\eta}}{3 \pi(1-T) I_{p}} \\
& \eta=I_{1} / I_{0},
\end{aligned}
$$

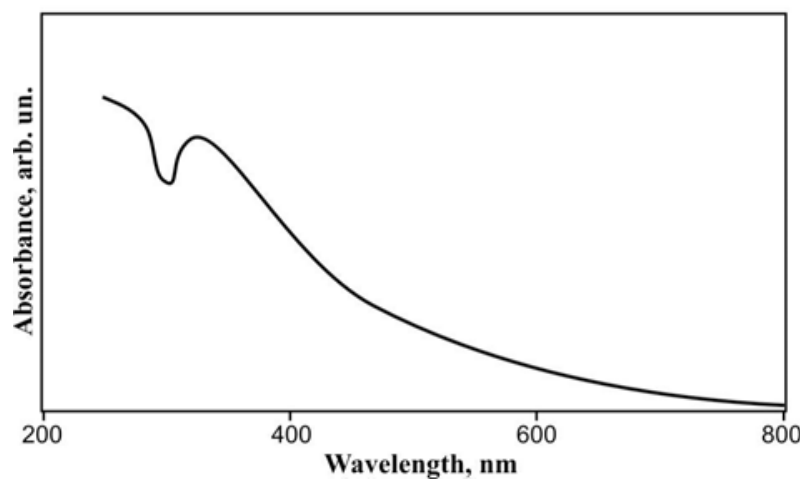

Fig. 2. UV-VIS optical absorption spectra of PbS quantum dots. 


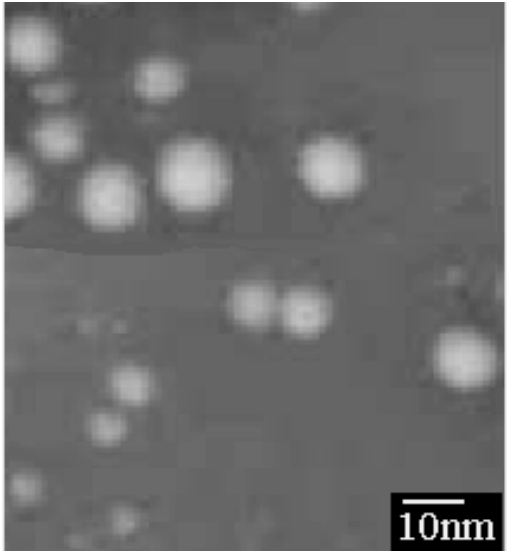

Fig. 3. TEM image of PbS quantum dots.

where $n$ is the linear refractive index, $c$ - the velocity of light, $\lambda$ - the measurement wavelength, $\alpha$ - the sample absorption coefficient at $\lambda, I_{p}-$ the pump intensity, $T-$ the transmission at $I_{p}, \varepsilon_{0}$ - the dielectric constant of the material. Here, $\eta$ is the diffraction efficiency, i.e., the intensity relation of the transmitted first order $\left(I_{1}\right)$ and zeroth order $\left(I_{0}\right)$ of the diffraction pattern, which appears by self-diffraction of a laser induced grating.

Using the experimental data, $n=1.52$ [21], $c=$ $=3 \times 10^{8} \mathrm{~m} / \mathrm{s}, \varepsilon_{0}=17.2$ for $\mathrm{PbS}[22], \lambda=632.8 \mathrm{~nm}, I_{p}=$ $=1.7 \times 10^{6} \mathrm{~W} / \mathrm{m}^{2}, \eta=0.05$ and $\alpha=6.65 \times 10^{6}$ [23], we calculate the value of $\chi^{3}$ as $19.6 \times 10^{-4} \mathrm{~m}^{2} / \mathrm{V}^{2}$. A comparison of this result with other published results could not be made because the discrepancy in the order of $\chi^{3}$ arises due to the smaller value of $I_{p}$, appearing in the formula used. For a high power laser with $I_{p} \approx \mathrm{GW} / \mathrm{m}^{2}$, the order will automatically come down. Since we have recorded the absorption onset at around $511 \mathrm{~nm}$, exciton resonance can not be the origin of the observed nonlinearity. Instead, we believe that the surface states absorption is the right mechanism to be considered here. However, the surface states are not detectable in the optical absorption spectra [24] may be due to a lower content of the surface states in the samples synthesized in the polymer matrix [25]. Here 'lower content' has only a relative meaning, the surface state in these nanoparticles is considerably higher than

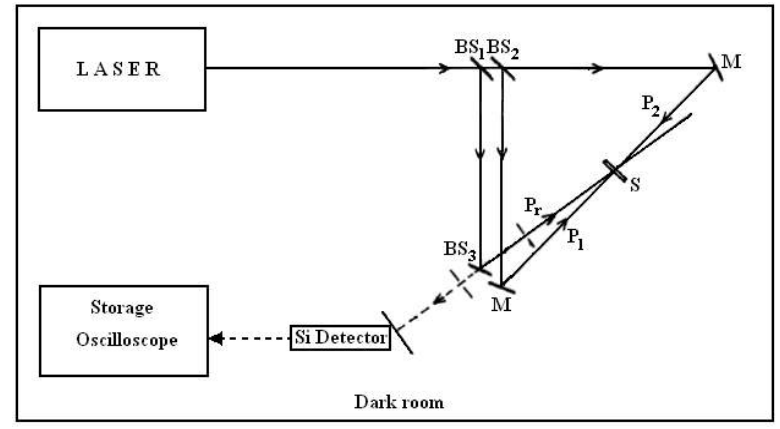

Fig. 4. Experimental set up for DFWM. those in the bulk material. The PL spectra of the sample also confirm the presence of surface states in our sample as described in the earlier work [15]. Thus, it is worth believing that the surface state absorption during intermixing of the laser beam on the sample may induce a transient change in the absorption coefficient resulting a change in the refraction index which in turn gives rise to nonlinearity in the sample.

\section{Conclusion}

$\mathrm{PbS}$ quantum dots with average size $10 \mathrm{~nm}$ has been synthesized through chemical route using polymer matrix (PVA). DFWM experiment was carried out using a low power continuous wave He-Ne laser. The result shows that the low power laser source can be used to study the nonlinear optical behavior of nanostructured materials.

\section{Acknowledgement}

We sincerely thank USIC, G.U and RSIC, NEHU for providing XRD and TEM, respectively.

\section{References}

1. R. del Coso, J. Requejo-Isidro, J. Solis, J. Gonzalo and C.N. Afonso // J. Appl. Phys. 95, p. 2755 (2004).

2. R. de Nalda, R. del Coso, J. Requejo-Isidro, J. Olivares, A. Suarez-Garcia, J. Solis, and C.N. Afonso // J. Opt. Soc. Amer. B 19, p. 289 (2002).

3. Y.G. Fuh Andy, C.Y. Huang, M.S. Tsai, J.M. Chen, and L.C. Chien // Chin. J. Phys. 33, p. 645 (1995).

4. M.J. Fernee, J. Warner, A. Watt, S. Cooper, N.R. Heckenberg, and R.H. Dunlop // Nanotechnology 15, p. 16 (2004).

5. Y. Wang and W. Mahler // Opt. Communs 61, p. 233 (1987)

6. T. Takada, J.D. Mackenzie, M. Yamane, K. Kang, N. Peyghambarian, R.J. Reeves, E.T. Knobbe, and R.C. Powell // J. Mater. Sci. 31, p. 423 (1996).

7. U. Woggon, Optical properties of Semiconductor quantum dot / Managing editor G. Höhler. Springer-Verlag, Berlin (1997) p. 177.

8. T. Yamaki, K. Asai, K. Ishigure, K. Sano, and K. Ema // Synth. Metals 103, p. 2690 (1999).

9. S.W. Lu, U. Sohling, M. Mennig, and H. Schmidt // Nanotechnology 13, p. 669 (2002).

10. P.A. Wolff, S.Y. Yuen, K.A. Harris, J.W. Cook, and J.F. Schetzina Jr. // Appl. Phys. Lett. 50, p. 1858 (1987).

11. F. Jochen, S. Jochen, R. Levyz, A. Martuccix and M. Guglielmix // Pure Appl. Opt. 6, p. 527 (1997).

12. Y. Yang, H. Fei, Z. Wei, Q. Yang, G. Sun, and L. Han // J. Luminescence, 66 \& 67, p. 133 (1996).

13. A. Kiessling, T. Baade, and L. Wenke // J. Mod. Opt. 43, p. 1525 (1996). 
14. P.A. Kurian and C. Vijayan // ISRS-2004, December 20-22, Chennai, India.

15. S. Chowdhury, D. Mohanta, G.A. Ahmed, S.K. Dolui, D.K. Avasthi, and A. Choudhury // J. Luminescence 114, p. 95 (2005).

16. S. Chowdhury, D. Mohanta, G.A. Ahmed, S.K. Dolui, D.K. Avasthi, and A. Choudhury // Nucl. Instrum. and Meth. B 240, p. 690 (2005).

17. D. Mohanta, S.S. Nath, A. Bordoloi, S.K. Dolui, and A. Choudhury // J. Appl. Phys. 92, p. 7149 (2002).

18. D. Mohanta, S.K. Dolui, and A. Choudhury // Ind. J. Phys. 75, p. 53 (2001).
19. S.B. Qadri, J.P. Yang, E.F. Skelton, and B.R. Ratna // Appl. Phys. Lett. 70, p. 1020 (1997).

20. S. Yang, S. Wang, and K.K. Fung // Pure Appl. Chem.72, p. 119 (2000).

21. J. Fick, J. Schell, R. Levy, A. Martucci, and M. Guglielmi // Pure Appl. Opt. 6, p. 527 (1997).

22. P.B. Littlewood // J. Phys. C: Solid State Phys. 12, p. 4459 (1979).

23. R.B. Schoolar, and J.R. Dixon // Phys. Rev. A 137, p. 667 (1965).

24. N. Chestnoy, T.D. Harris, R. Hull, and L.E. Brus // J. Phys. Chem. 90, p. 3393 (1986).

25. W. Chen, Z. Wang, Z. Lin, and L. Lin // J. Appl. Phys. 82, p. 3111 (1997). 